\title{
Micro-Fourier-Transform Infrared Reflectance Spectroscopy as Tool for Probing IgG Glycosylation in COVID-19 Patients
}

\section{Carla Bandeira}

Universidade Federal do ABC

Karen Madureira

Instituto de Infectologia Emílio Ribas

Meire Rossi

Instituto de Infectologia Emílio Ribas

Juliana Gallo

Instituto de Infectologia Emílio Ribas

Ana Paula da Silva

Instituto de Infectologia Emílio Ribas

Vilanilse Torres

Instituto de Infectologia Emílio Ribas

Vinicius de Lima

Universidade de São Paulo

Norival Kesper Júnior

Universidade de São Paulo

Janete Almeida

São Paulo State University

Rodrigo Zerbinati

Universidade de São Paulo

Paulo Braz-Silva

Universidade de São Paulo

Jose Angelo Lauletta Lindoso

Universidade de São Paulo

Herculano Martinho ( $\square$ herculano.martinho@ufabc.edu.br)

Universidade Federal do ABC

\section{Research Article}

Keywords: Micro-Fourier-Transform, Infrared Reflectance Spectroscopy, Probing IgG Glycosylation, COVID19 Patients 
Posted Date: September 29th, 2021

DOl: https://doi.org/10.21203/rs.3.rs-916636/v1

License: (c) (i) This work is licensed under a Creative Commons Attribution 4.0 International License. Read Full License

Version of Record: A version of this preprint was published at Scientific Reports on March 11th, 2022. See the published version at https://doi.org/10.1038/s41598-022-08156-6. 


\section{Micro-Fourier-Transform Infrared Reflectance spectroscopy as tool for probing IgG glycosylation in COVID-19 patients}

Carla Carolina Bandeira ${ }^{1}$, Karen Cristina Rolim Madureira² ${ }^{2}$ Meire Bocoli Rossi ${ }^{2}$, Juliana Failde

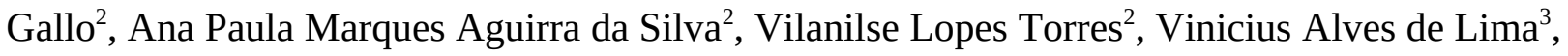
Norival Kesper Júnior ${ }^{3}$, Janete Dias Almeida ${ }^{4}$, Rodrigo Merlim Zerbinati ${ }^{5}$, Paulo Henrique BrazSilva $^{5,6}$, José Angelo Lauletta Lindoso ${ }^{2,3,7}$, Herculano da Silva Martinho ${ }^{{ }^{*}}$

\footnotetext{
${ }^{1}$ Universidade Federal do ABC, Centro de Ciências Naturais e Humanas, Av. dos Estados, 5001, 09210-580, Santo André, SP, Brazil

${ }^{2}$ Instituto de Infectologia Emilio Ribas - Secretaria de Estado da Saúde, São Paulo, Brazil.

${ }^{3}$ Laboratório de Protozoologia (LIM-49-HC-FMUSP), Instituto de Medicina Tropical de São Paulo, Faculdade de Medicina da Universidade de São Paulo -FMUSP, São Paulo, Brazil.

${ }^{4}$ São Paulo State University (Unesp), Institute of Science and Technology, São José dos Campos, Department of Biosciences and Diagnosis.

${ }^{5}$ Laboratório de Virologia (LIM-52-HC-FMUSP), Instituto de Medicina Tropical de São Paulo, Faculdade de Medicina da Universidade de São Paulo - FMUSP, São Paulo, Brazil

${ }^{6}$ Departamento de Estomatologia, Faculdade de Odontologia da Universidade de São Paulo - FOUSP, São Paulo, Brazil

${ }^{7}$ Departamento de Moléstias Infecciosas e Parasitárias, Faculdade de Medicina da Universidade de São Paulo - FMUSP, São Paulo, Brazil
}

*Corresponding Author: herculano.martinho@ufabc.edu.br 


\begin{abstract}
It has been reported that patients diagnosed with COVID-19 become critically ill primarily around the time of activation of the adaptive immune response. However the role of antibodies in the worsening of disease is not obvious. Higher titers of anti-spike immunoglobulin IgG1 associated to low fucosylation of the antibody Fc tail have been associated to excessive inflammatory response. In contrast it has been also reported in literature that NP-, S-, RBD- specific $\operatorname{IgA}, \operatorname{IgG}, \operatorname{IgM}$ are not associated with SARS-CoV-2 viral load, indicating that there is no obvious correlation between antibody response and viral antigen detection. In the present work the micro-Fourier-Transform Infrared reflectance spectroscopy (micro-FTIR) was employed to investigate blood serum samples of healthy and COVID-19 (mild or oligosynthomatic) individuals (82 healthcare workers volunteers in "Instituto de Infectologia Emilio Ribas", São Paulo, Brazil). The molecular-level-sensitive, multiplexing quantitative and qualitative FTIR data probed on $1 \mathrm{~mL}$ of dryed biofluid was compared to Signal-to-Cutoff index of chemiluminescent immunoassays CLIA and ELISA (IgG antibodies against SARS-CoV-2). Our main result indicated that $1702-1785 \mathrm{~cm}^{-1}$ spectral window (carbonyl $\mathrm{C}=\mathrm{O}$ vibration) appeared to be a spectral marker of the degree of $\mathrm{IgG}$ glycosylation, allowing to probe distinctive subpopulations of COVID-19 patients, depending on their degree of severity. The specificity was $87.5 \%$ while the detection rate of true positive was $100 \%$. The computed area under the receiver operating curve was equivalent to CLIA, ELISA and other ATR-FTIR methods (>0.85). In summary, overall discrimination of healthy and COVID-19 individuals and severity prediction as well could also be potentially implemented using micro-FTIR reflectance spectroscopy on blood serum samples. Considering the minimal and reagent-free sample preparation procedures combined to fast (few minutes) outcome of FTIR we can state that this technology is very suitable for fast screening of immune response of individuals with COVID-19. It would be an important tool in prospective studies, helping investigate the physiology of the asymptomatic, oligosymptomatic, or severe individuals and measure the extension of infection dissemination in patients.
\end{abstract}




\section{INTRODUCTION}

In 2003, severe acute respiratory syndrome coronavirus (SARS-CoV) caused a devastating worldwide outbreak with a mortality rate of 10\%[1]. In December 2019, a SARS-CoV-like coronavirus, novel coronavirus 2019 (SARS-CoV-2), emerged from Hubei Province in China and quickly spread to mainland China and other parts of the world[2]. As of September 27, 2021, there have been more than 231 million confirmed cases of COVID -19, including nearly 5 million deaths. More than 5 million doses of vaccine have been administered, according to WHO [3] .

Accumulating evidence suggests that a subset of patients with severe COVID -19 may have a cytokine storm syndrome[4]. It has been recommended that hyperinflammation should be recognized and treated using already approved therapies with proven safety profiles to reduce the increasing mortality[4]. Ruan et al.[5] presented as predictors of mortality elevated ferritin (mean 1,297.6 ng/ml in non-survivors versus $614.0 \mathrm{ng} / \mathrm{ml}$ in survivors; $\mathrm{p}<0.001)$ and IL-6 ( $\mathrm{p}<0.0001)$, suggesting that mortality may be due to viral-induced hyperinflammation[5]. Mehta et al. [4] suggested that all patients with severe COVID -19 should be screened for hyperinflammation based on laboratory trends (e.g., increasing ferritin, decreasing platelet counts or erythrocyte sedimentation rate) and HScore [6] (see table in [4] ) to identify the subset of patients in whom immunosuppression might improve mortality.

Antibodies or immunoglobulins (IgG) are glycoproteins that make up the humoral part of the adaptive immune system and fight pathogens such as viruses, bacteria, parasites, and diseased cells [7]. Due to their immense variability, there are more than 10 billion types of plasma antibodies, which can be divided into four subclasses: IgG1, IgG2, IgG3 and IgG4 [8] in order of decreasing abundance in serum [9]. IgG1, for example, reacts primarily to soluble and membrane protein antigens [10]. 
It has been noticed that patients diagnosed COVID -19 become severely ill, especially at the time of activation of the adaptive immune response[11-13]. Hoepel et al. [11] reported that antibodies play a role in disease exacerbation at the time of seroconversion. They have shown that in the early phase of severe acute respiratory illness SARS-CoV-2 spike protein-specific IgG in the serum of critically ill COVID -19 patients triggers an excessive inflammatory response by human alveolar macrophages. They found that this excessive inflammatory response depends on two antibody features specific to patients with severe COVID -19: i) higher titers of anti-spike IgG and ii) the anti-spike IgG of patients with severe COVID -19 is inherently more pro-inflammatory due to different glycosylation, particularly low fucosylation, of the Fc tail of the antibody. Remarkably, the low fucosylation of the anti-spike IgG normalized within a few weeks after initial infection with SARS-CoV-2, suggesting that the increased antibody-dependent inflammation occurs mainly at the time of seroconversion. Analogously Chakraborty et al. [12] suggested that patients with severe COVID -19 have a unique serological signature, including an increased likelihood of IgG1 with afucosylated Fc glycans. After analyzing 77 patients (ICU, inpatient, outpatient, and pediatric), they concluded that Fc modification on coronavirus IgGs of severe acute respiratory syndrome increased interactions with the activating Fcy receptor FcyRIIIa. When incorporated into immune complexes, Fc afucosylation increased the production of inflammatory cytokines by monocytes, including IL-6 and tumor necrosis factor (TNF). These results show that disease severity in COVID -19 correlates with the presence of pro-inflammatory IgG-Fc structures, including afucosylated IgG1 [12] . On the other hand, Luo et al. [13] showed that NP -, S-, RBD- specific IgA, IgG, IgM were not associated with SARS-CoV-2 viral load, suggesting that there is no obvious correlation between antibody response and viral antigen detected in nasopharyngeal swabs [13]. They analyzed antibody and cytokine responses in COVID -19 from asymptomatic to severe patients (123 serum samples from 63 COVID -19 patients) and evaluated the impact of various risk factors, including comorbidities, 
male sex, and advancing age, on the host immune response COVID -19 patients. These antagonistic data highlight the relevance of investigating structural aspects of serum IgG from COVID -19 patients to establish its rule as a severity marker.

Vibrational spectroscopic techniques such as Fourier-Transform Infrared Absorption (FTIR) have been successfully used to study biological samples. They provide quantitative and qualitative multiplexed information at the molecular level, even when triggered by subtle changes in a sample [14]. Vibrational spectroscopy involves label-free techniques that allow the detection of electronic changes in the internal vibrational energy levels of biomolecules. This technique has been used in the study of cells, tissues and biofluids and provided valuable insights into pathologies[15-18]. Barauna et al. [19] studied saliva samples from throat swabs using Attenuated Total Reflectance FTIR (ATRFTIR). Initial classification of swab samples into negative and positive COVID -19 infections was based on symptoms and PCR results ( $\mathrm{n}=111$ negatives and 70 positives). A blind sensitivity of 95\% and a specificity of $89 \%$ were achieved. Artificial saliva samples containing inactivated, $\gamma$-irradiated COVID -19 virus particles at concentrations up to 1582 copies/ml were also analyzed to understand the spectral response of the virus. Kitane et al. [20] reported a method for COVID -19 discrimination based on spectral ATR-FTIR analysis of 280 RNA extracts from nasopharyngeal samples (100 SARS-CoV-2 PCR-positive patients and 180 SARS-CoV-2 PCR-negative patients). The proposed method is based on RNA extraction followed by ATR-FTIR analysis of the RNA extracts and machine learning modeling. The authors report that this technique achieves $97.8 \%$ accuracy, $97 \%$ sensitivity, and $98.3 \%$ specificity while reducing the testing time from hours to minutes after RNA extraction. The reported area under the AUC ranged from 0.54 to 1 , depending on the statistical model used. Wood et al. [21] proposed a portable infrared spectrometer with custom-made transflectance accessories for rapid point-of-care detection of COVID -19 markers in saliva. They tested the system on samples from 29 subjects who tested positive for SARS-CoV-2 by RT -qPCR 
and 28 who were negative, and achieved a sensitivity of $93 \%$ and a specificity of $82 \%$. This work did not aim to explore the rich biochemical information contained in the spectral data. In particular, FTIR has been used to investigate the secondary structural composition and changes in structural dynamics of IgG upon glycation, oxidation and glycoxidation [22]. Thus, it is a suitable technology to study the structural changes of IgG by COVID -19.

To clarify this point, we performed an FTIR study of blood serum from adult healthcare workers with asymptomatic or mild acute SARS-CoV-2 infection compared to healthy individuals, all tested against anti-IgG for SARS-Cov-2, using a microscope-based version of the technique and specific methodology. Detailed characterization of the immune response of asymptomatic or oligosymptomatic individuals is important for prospective studies to describe the physiology of these cases compared to cases with higher severity and also to determine the extent of spread of infection by these patients.

\section{METHODS}

\subsection{Sampled population}

Serum samples from 82 healthcare workers volunteers of the "Instituto de Infectologia Emilio Ribas”, São Paulo, Brazil were included in this study. These samples are part of a cohort study to evaluate the seroprevalence of SARS-CoV-2 infection in healthcare population. Samples were collected from July to November, 2020, before the beginning of the vaccination program in Brazil. All patients were asymptomatic or oligosympthomatic. This study was conducted in accordance with the Declaration of Helsinki, and the protocol was approved by Research Ethics Committee of the “Instituto de Infectologia Emílio Ribas”, São Paulo, Brazil, protocol number CAAE 32264120.5.2001.0061. The invited volunteers were informed about the objectives, propositions and conditions of this project, and those who agreed to participate in the research signed the free and informed consent term. A volume of $5 \mathrm{ml}$ of whole blood was collected by peripheral 
vein puncture and stored in a $10 \mathrm{ml}$ dry tube for each patient. Then it was centrifuged at $2052 \mathrm{~g}$ for 10 minutes to separate the serum. Two $\mathrm{mL}$ of serum were stored in a $-20{ }^{\circ} \mathrm{C}$ freezer until performing the immunoassays and FTIR test.

\subsection{Detection of antibodies anti-SARS-CoV-2}

\subsubsection{Chemiluminescent immunoassay (CLIA)}

A CLIA (IgG Antibodies against SARS-CoV-2; reagents pack \# 619 9919; VITROS Imumunodiagnostic calibrator \# 619 992; Ortho Clinical Diagnostics, Raritan, NJ, USA) immunoassay was performed to detect immunoglobulin G anti-Spike protein from SARS-CoV-2. This assay does not differentiate binding IgG antibodies from virus-neutralizing IgG antibodies[23]. The results were expressed in terms of ratio of the sample signal to a calibrator-assigned cutoff signal with threshold of 1.0. Negative diagnosis were considered for outcomes with reactivity index $<1$ otherwise they were considered positive[24]. The test sensitivity was reported to be $90.0 \%$ ( $\geqslant 8$ days) and specificity of $100.0 \%$ [24]. This serie of CLIA diagnostic test uses S antigens for SARSCoV-2 detection[25].

\subsubsection{Enzyme Linked Immuno Sorbent Assay (ELISA)}

Anti-SARS-CoV-2 IgG ELISA (Euroimmun Medizinische Labordiagnostika, Lübeck, Germany; \# EI 2606-9601 G; Indirect ELISA) assay was performed to detect IgG antibodies against SARS-CoV2. In this diagnostic test IgG antibodies against SARS-CoV-2 spike protein subunit 1 (S1) are detected in human serum or plasma. Following the instructions of the manufactures, serum samples were 1:101 diluted, added to wells coated with recombinant SARS-CoV-2 antigen and incubated for 60 minutes at $37{ }^{\circ} \mathrm{C}$. Then wells were washed three times and followed by the addition of HRPconjugated anti-human IgG and subsequent incubation for 30 minutes at $37^{\circ} \mathrm{C}$. Wells were washed three times and a chromogen solution was added. After 30 minutes of incubation at room temperature, the reaction was stopped and the absorbance at $450 \mathrm{~nm}$ with reference at $620 \mathrm{~nm}$ was 
read on a microplate reader. A ratio between the extinction of the sample and calibrator on each plate were calculated. According to recommendations of manufacturer, a signal-to-cutoff ratio smaller than 0.8 is considered negative, while a positive one if greater than 1.1. The borderline region falls into 0.8-1.1 interval. The sensitivity and specificity of this assay were reported to be $90.0 \%$ and 100\%, respectively[25].

\subsection{Fourier-Transform Infrared Absorption (FTIR) spectroscopy}

All samples were brought to room temperature prior to preparation for FTIR measurements. Aliquots of $1 \mu \mathrm{L}$ of serum (1:3 in ultrapure water) were deposited in platinum sample holder and dried at $80 \%$ relative moisture under a desicator with $\mathrm{NaCl}$ saturated solution in order to avoid cofee ring effect and obtain a homogeneous biofilm[26]. The final droplet presented an average radius of 1000 +/- $150 \mu \mathrm{m}$. A Varian 610 FT-IR Micro-spectrometer coupled to a 640-IR FT-IR spectrometer (two dimensional LN2 Ge detector) was used in reflectance mode for spectra acquisition. The window for spectra acquisition was $150 \times 150 \mu \mathrm{m}^{2}$, while the spectral resolution was set to $2 \mathrm{~cm}^{-1}$. The number of scans was 32 per sample (equivalent to 30s time of acquistion). The summarized scheme of methodology is shown on Fig. 1.

\subsection{Statistical Analysis}

\subsubsection{Principal Components Analysis (PCA)}

The classical Principal Components Analysis (PCA)[27] was performed on mean centered raw data to extract outliers and identify possible experimental bias. All spectral analysis steps were implemented in the ChemSpec vignette available in the software R[28] . Outliers were identied using the $Q$ and $T^{2}$ Hotelling's statistics. The $Q$ statistics indicates how well each observation matches to the PCA model and the Q-residuals measure the residual between a sample and its projection on the factors retained in the model. Large residual outliers can be detected by inspection of $Q$ residuals. On the other hand, Hotelling's $T^{2}$ value represents a measure of the variation in each sample within the 
model, indicating how far each sample is from the center (scores $=0$ ) of the model. It is a quantifier for scores outliers. The $T^{2}$ Hotelling's versus $Q$-residuals (reduced) plot were inspected raw spectral data.

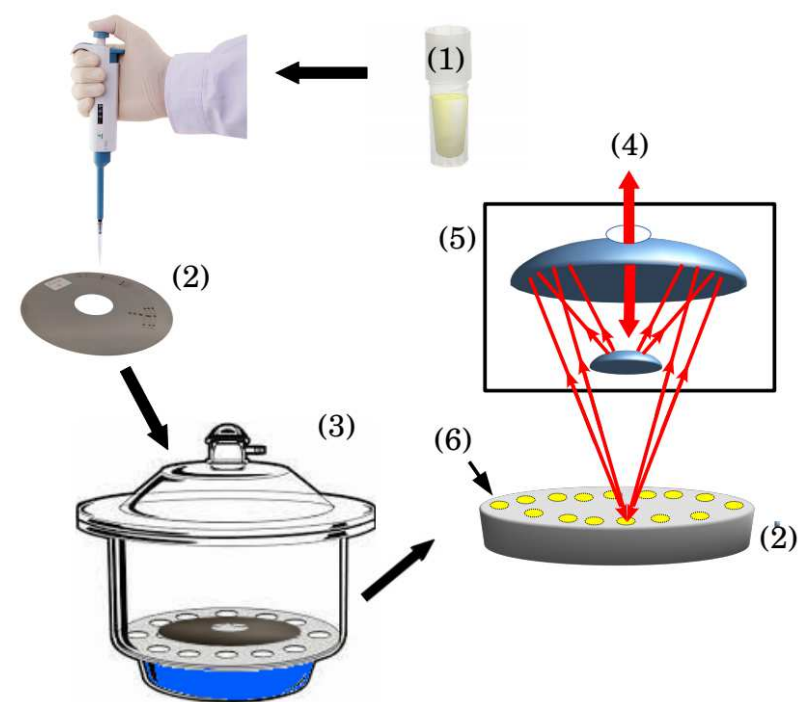

Figure 1. Scheme COVID-19 patient serum micro-FTIR reflectance measurements. One $\mu \mathrm{L}$ of diluted (1:3 in ultra-pure water) serum sample solution (1) was transferred to circular platinum sample holder (2). Then the sample holder was installed in a desiccator with saturated solution of $\mathrm{NaCl}$ (3) for drying in 10 min. After this period the sample holder was installed on the FTIR reflectance accessory of the micro-FTIR spectrometer. The IR beam (4) passing through the IR lens (5) focuses the light on a given aliquot of sample (6). The reflected light is collected by same lens and analyzed by the spectrometer.

\subsubsection{Partial Least Squares - Discriminant Analysis (PLS-DA)}

All spectra were pre-processed to become comparable for statistical analysis. The baseline was corrected using the least-squares polynomial curve fitting method as described by Lieber and Mahadevan-Jansen[29] . All spectra were normalized and scaled using Probabilistic Quotient Normalization [30] .

Then PLS-DA analysis was performed. PLS is a multivariate supervised method that uses linear regression of original variables to predict the class membership. In our case the PLS regression was performed using the plsr function provided by $\mathrm{R}$ pls package [28; 31]. The classification and cross-validation were performed using the corresponding wrapper function using the caret package[28]. A permutation test was performed to assess the performance of class 
discrimination. In each permutation, a PLS-DA model was built between the data and the permuted class labels using the optimal number of components determined by leave-one-out cross validation for the model based on the original class assignment. The class discrimination performance was measured using classification accuracy, $R^{2}$, and $\mathrm{Q}^{2}$ parameters. The first one is based on prediction accuracy. The $\mathrm{R}^{2}$ parameter is the "goodness of fit" or explained variation which is based on the ratio of the between group sum of the squares and the within group sum of squares. On the other hand, $Q^{2}$ is the "goodness of prediction", or predicted variation, calculated from cross validation. In each round, the predicted data are compared with the original data, and the sum of squared errors is calculated and then summed over all samples (Predicted Residual Sum of Squares or PRESS). For convenience, the PRESS is divided by the initial sum of squares and subtracted by 1 to scale to $R^{2}$. Good predictions will have low PRESS or high $Q^{2}$ while negative $Q^{2}$ means the model is not predictive at all or is overfitted[18; 32; 33] .

Two quantifiers were used to measure the vibrational band frequency importance in PLS-DA model. The first, Variable Importance in Projection (VIP) is a weighted sum of squares of the PLS loadings taking into account the amount of explained spectral intensity-variation in each dimension. The other importance measure is based on the weighted sum of PLS-regression. The weights are a function of the reduction of the sums of squares across the number of PLS components. For multiple-group analysis, the same number of predictors will be built for each group and the average of the feature coefficients were used to indicate the overall coefficient-based importance.

The receiver operating characteristic (ROC) analysis was used to evaluate the discriminating performance and the area under the ROC curve (AUC) as its summary index. In general tests with excellent discriminating capability will furnish AUC > 0.80[34; 35].

\subsubsection{Normality and F tests}


The Kolmogorov-Smirnov test of normality[36] was applied to test the hypothesis that IgG data does not differ significantly from that which is normally distributed. The F-test was used to test the hypothesis of equality of averages of two sets of data populations of unequal size (IgG and demographic data)[37] . 
Table 1. Demographic data of volunteers, a set of 82 healthcare workers from "Instituto de Infectologia Emilio Ribas”, São Paulo, Brazil.

\begin{tabular}{|c|c|c|c|}
\hline Variable & $\begin{array}{c}\text { Positive } \\
(n=33)\end{array}$ & $\begin{array}{c}\text { Negative } \\
(n=49)\end{array}$ & p-value \\
\hline \multicolumn{4}{|l|}{ Gender } \\
\hline Female & $76.7 \%$ & $72.1 \%$ & $<5 \%$ \\
\hline Male & $23.3 \%$ & $27.9 \%$ & $<5 \%$ \\
\hline Age & $44 \pm 14$ & $49 \pm 12$ & $<5 \%$ \\
\hline Comorbidities $^{1}$ & $39.4 \%$ & $16.3 \%$ & $45.4 \%$ \\
\hline$\overline{\text { BMI }^{2}}$ & $25 \pm 12$ & $30 \pm 12$ & $<5 \%$ \\
\hline
\end{tabular}

${ }^{1}$ rheumatoid arthritis, asthma, diabetes, systemic arterial hypertension, chronic obstructive pulmonary disease, obesity, hypothyroidism. ${ }^{2} B M I=$ body mass index, defined as ratio of weight $(\mathrm{kg})$ and squared height $(\mathrm{m})$.

\section{RESULTS AND DISCUSSION}

The main clinical pieces of information of volunteers is presented on Table 1. Percentages of 40.2\% ( $\mathrm{n}=33)$ and $59.8 \%(\mathrm{n}=49)$ of individuals tested positive and negative, respectively. Female individuals predominated on the sampled population representing $>72 \%$ of total patients in both positive and negative groups. However we notice that this unbalance did not represent a bias on the positive or negative rates within confidence value of 5\%. The average age of patients in positive and negative groups was $44 \pm 14$ years and $49 \pm 12$ years, respectively. Again these differences were not statistically relevant for COVID-19 diagnostic purposes. Likewise the body mass index (BMI) did not represent a statistically relevant variable comparing negative and positive groups. On the other hand, the presence of comorbidities presented a well-defined increased risk for positive test against COVID-19. A percentage of $39.4 \%$ of individuals which tested positive presented some kind of comorbidity (rheumatoid arthritis, asthma, diabetes, systemic arterial hypertension, chronic obstructive pulmonary disease, obesity, or hypothyroidism) while this rate is only $16.3 \%$ in the negative group. The outcome of F-test indicated that there is enough evidence against the hypothesis that population sampled with positive and negative test has the same average $(p=45.4 \%)$. The correlation between comorbidities and prevalence of COVID-19 is well reported on literature and 
our findings give one more piece of evidence concerning this important aspect of etiology of COVID-19[38] . We notice that CLIA and ELISA tests were 100\% concordant about the patients diagnosis in our cohort.

The average FTIR spectra for the negative (black line) and positive (red line) classes of samples in the fingerprint spectral window are shown on Fig. 2a). Assignment based on refs. [22; 3941] for the relevant vibrational bands is shown in Table 2. Proteins, amino acids, and nucleic acids vibrational bands dominate the spectra in accordance to the average chemical composition of the blood serum [42].
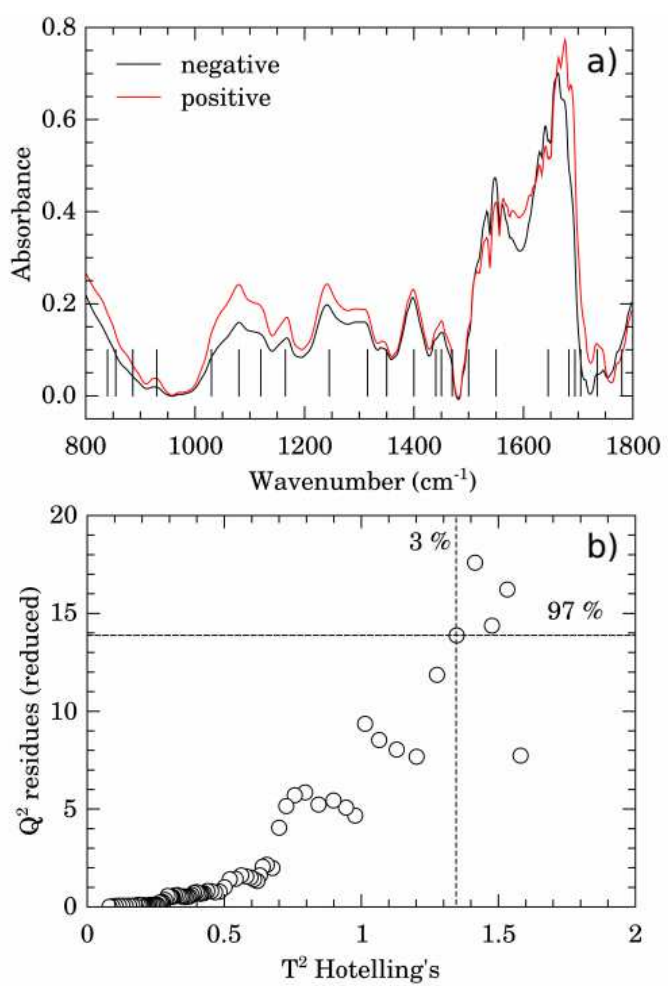

Figure 2. a) Average FTIR blood serum spectra for negative (black line) and positive (red line) groups. The vertical lines represents the main vibrational bands contributing to discrimination of groups. b) Outliers identification by inspection of Q residuals (reduced) versus Hotelling's. The outliers were indicated by “*”. Dashed lines horizontal and vertical lines represents confidence limit of 3\% (Hotelling's $\mathrm{T}^{2}$ ) and 97\% (Q residuals), respectively.

Prior to data processing and multivariate statistical analysis, a quality check evaluation was performed on raw spectral data to identify anomalous spectra, outliers and/or biased patterns. The PCA was calculated on mean-centered raw data and the $Q$ residuals (reduced) versus $\mathrm{T}^{2}$-Hotelling's plot [18; 33] was checked (Fig. 2b) in order to find residuals and scores outliers. Data outside of the 
confidence limits of $97 \%$ for scores and $3 \%$ for residuals were considered outliers (indicated by “*”, in Fig. 2b) and removed in further analysis.

Table 2 - Assignment of vibrational modes, based on refs. [22;39-41].

\begin{tabular}{|c|c|}
\hline Wavenumber $\left(\mathrm{cm}^{-1}\right)$ & Assignment \\
\hline $840-845$ & Left-handed helix DNA (Z form) \\
\hline 850-856 & $\mathrm{C}_{2}$ ' endo/anti of deoxyribose in B-form helix conformation \\
\hline 886 & C-C, C-O deoxyribose \\
\hline 930 & Left-handed helix DNA (Z form) \\
\hline 1030 & Stretching C-O ribose \\
\hline 1080 & Ring stretching vibrations in phenylalanine, tryptophan or tyrosine \\
\hline 1120 & Symmetric stretching P-O-C, phosphorylated saccharide residue \\
\hline 1165 & C-O stretching mode of C-OH groups of serine, threonine, tyrosine \\
\hline 1245 & Amide III $\alpha$-helix conformation of proteins \\
\hline 1315 & Amide III of proteins \\
\hline 1350 & Stretching C-O, deformation C-H, deformation N-H \\
\hline 1400 & Symmetric stretching vibration of COO- group of fatty acids and amino acids \\
\hline 1440 & Stretchinh C-H in polysaccharides, pectin \\
\hline 1450 & Asymmetric $\mathrm{CH}_{3}$ bending in proteins \\
\hline 1470 & $\mathrm{CH}_{2}$ bending vibration in lipids and proteins \\
\hline 1500 & $\begin{array}{l}\text { In-plane } \mathrm{CH} \text { bending vibration from the phenyl ring in phenylalanine, tryptophan } \\
\text { or tyrosine }\end{array}$ \\
\hline $1515-1580$ & Amide II of proteins \\
\hline $1630-1665$ & $\beta$-sheet structure of Amide I of proteins \\
\hline 1683 & Unordered random coils and turns of Amide I of proteins \\
\hline 1689-1698 & $\beta$-sheet structure of Amide I of proteins \\
\hline $1700-1708$ & $\mathrm{C}=\mathrm{O}$ in thymine \\
\hline 1735 & $\mathrm{C}=\mathrm{O}$ in polysaccharides \\
\hline $1768-1786$ & $\mathrm{C}=\mathrm{O}$ in IgG carbonyl group - glycosilation \\
\hline
\end{tabular}

PLS-DA analysis was then performed on processed, normalized and scaled spectra. The optimal number of factors was determined by cross-validation after inspection of accuracy, $\mathrm{R}^{2 \text {, }}$ and $\mathrm{Q}^{2}$. Figure 3 presents the pairwise scores up to $5^{\text {th }} \mathrm{PC}$ (4.7\% of explained variance) for positive and negative groups. 


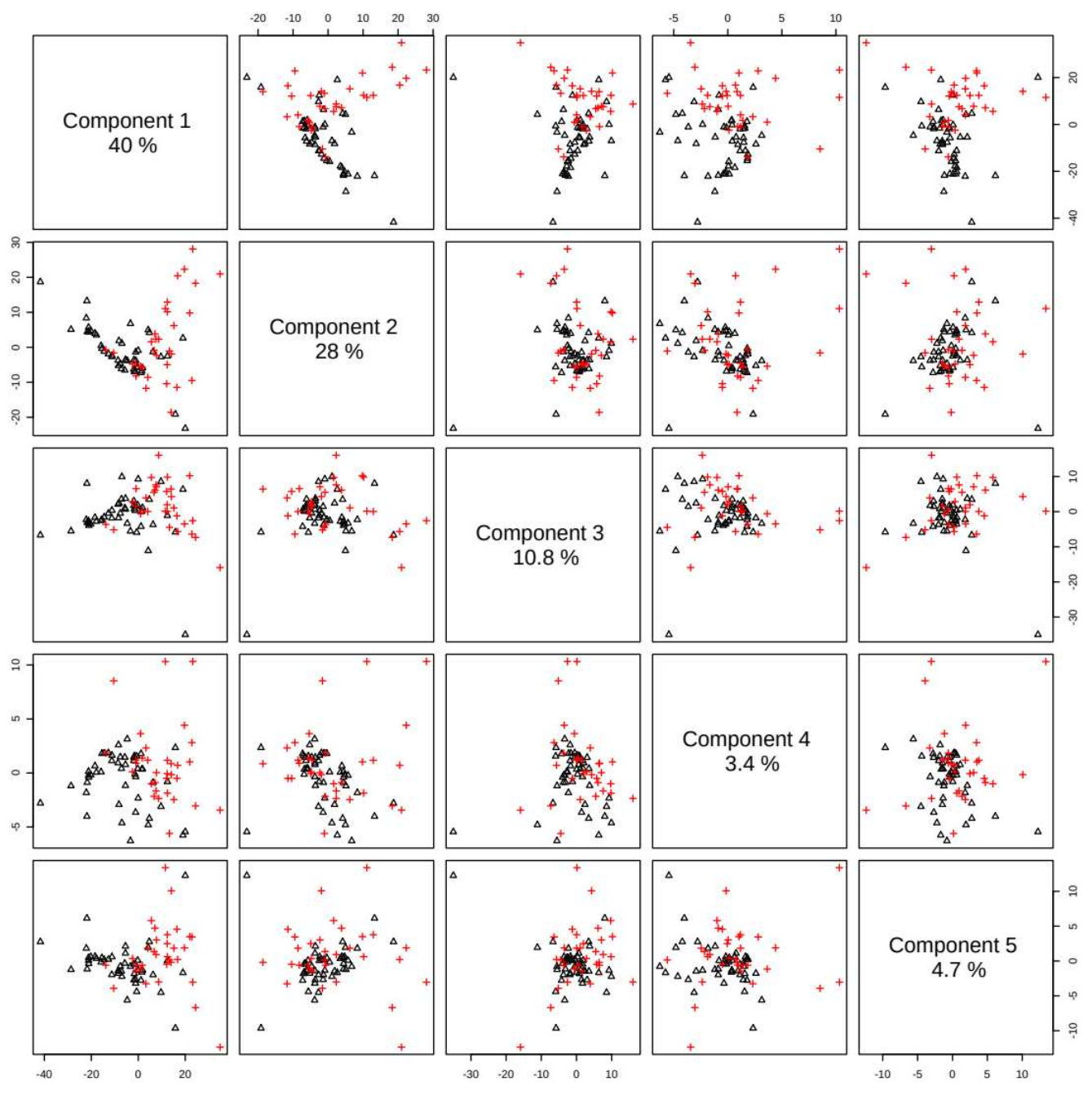

Figure 3. Pairwise score plots between the selected PLS-DA components. The explained variance of each component is shown in the corresponding diagonal cell. ( +: positive, $\Delta$ : negative)

At first glance, combinations including components 1 and 2 are prone to discriminate positive and negative samples. In fact, the best performance of PLS-DA classification as observed considering 2 components (Fig. 4a). In this case the observed accuracy on groups discrimination was $76 \%$ while $\mathrm{R}^{2}=0.39$ and $\mathrm{Q}^{2}=0.34$. 

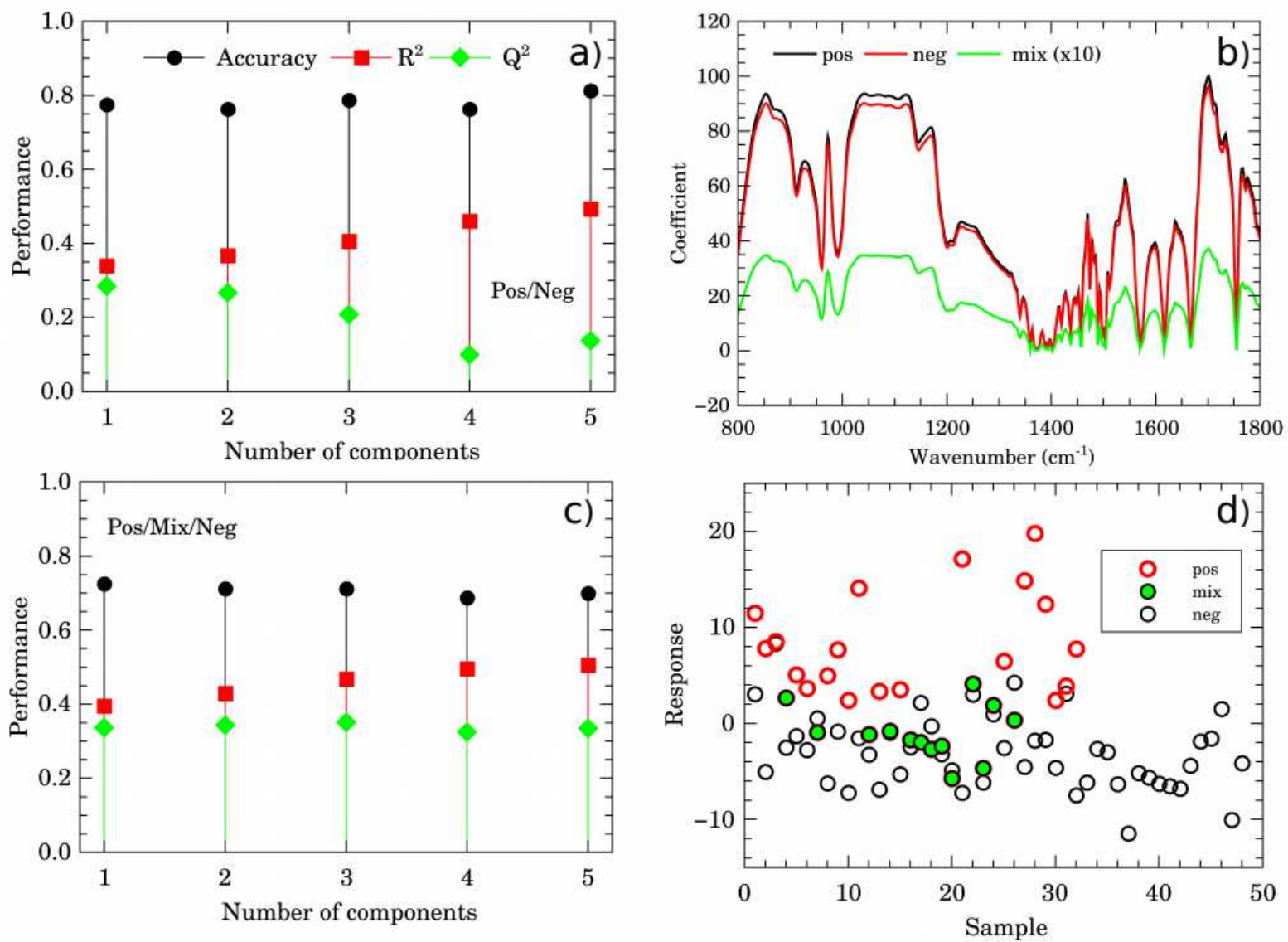

Figure 4. PLS-DA classification performance using different number of components following accuracy, $\mathrm{R}^{2}$ and $\mathrm{Q}^{2}$ criteria for two (positive/negative, a) and three (positive/mixture/negative, c) groups. The regression coefficient and calculated response in PLS-DA for sample classes are shown in $\mathbf{b}$ ) and d), respectively.

The regression coefficients are shown in Fig. 4b). Both curves for discriminating positive and negative groups appeared smooth, showing no random fluctuations around positive and negative values, which would be a symptom of overfitting. However, the coefficients for positive and negative classes appeared superimposed in many spectral windows, indicating greater similarity among them. The calculated response is shown in Fig. 4d). Sensibility and specificity were 53.1\% and $\mathbf{8 7 . 5 \%}$, respectively in this case. Interestingly, there is a distinct subgroup of misclassified samples labeled as a mixing group. This subgroup showed a clear spectral signature, as seen in the heatmap in Fig. 5. 


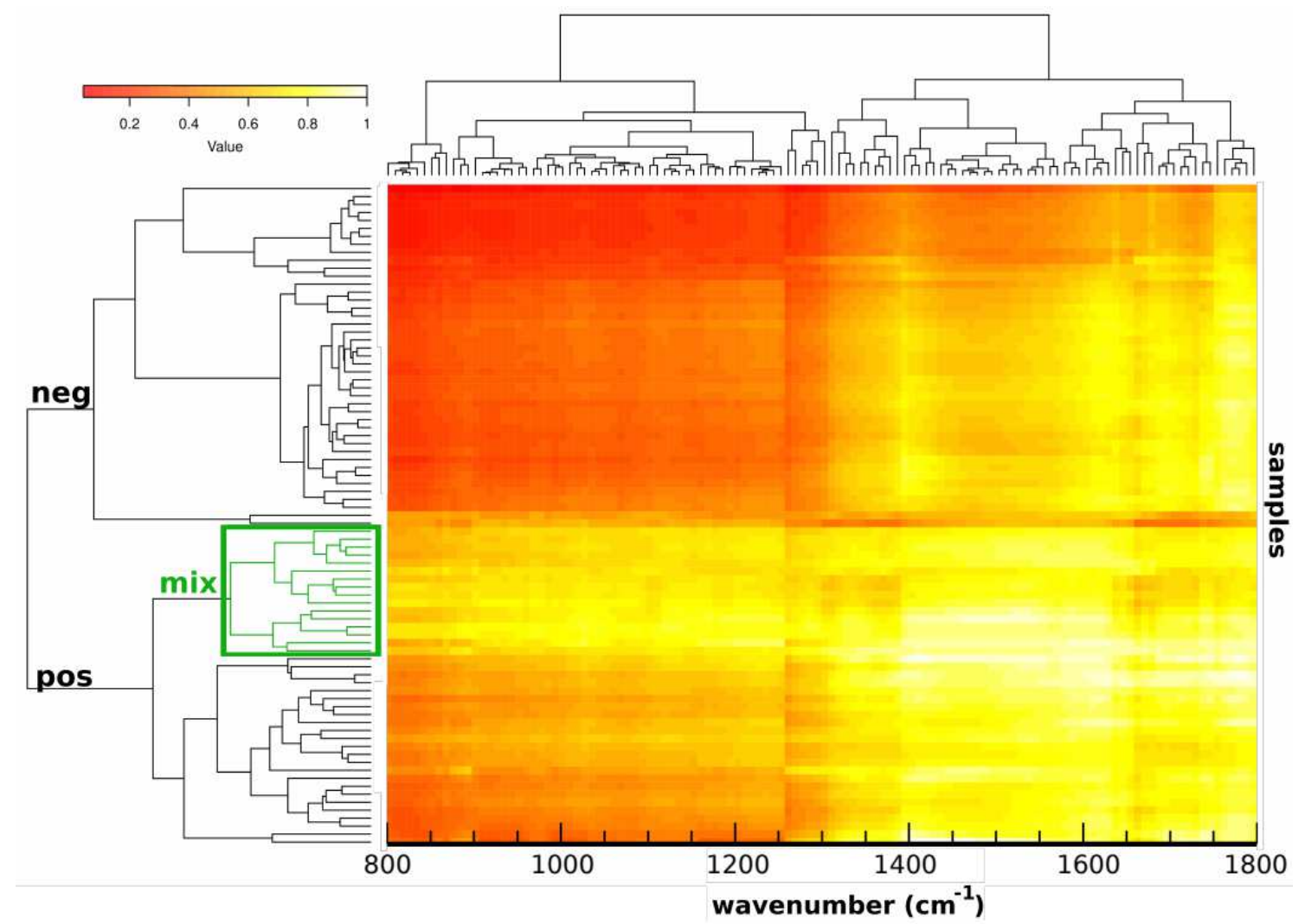

Figure 5. Clustering result shown as heatmap organized by samples (vertical axis) and wavenumber (horizontal axis). Groups negative, positive and the mix grouped into distinct clusters.

We recalculated the PLS-DA model including 3 possible groups (positive, negative, and mix). The performance of the model classification is shown in Fig. 4c). $\mathrm{R}^{2}$ and $\mathrm{Q}^{2}$ presented a consistent increase of $\sim 0.1$ while the accuracy was around $76 \%$. The coefficient and response obtained are indicated in green in Figs $4 \mathrm{~b}$ ) and $4 \mathrm{~d}$ ), respectively. Specificity was 87.5 while the detection rate of true positive and true mix increased to $100 \%$.

However, one outstanding result was observed contrasting the IgG Signal-to-Cutoff index from CLIA of each positive, mix, and negative group to FTIR. Histograms in Fig. 6a)-c) summarizes this finding. The positive group presented a broad IgG reactivity index distribution between 1 and 17 while the mix group presented reactivity ranging from 1 to 8 . 

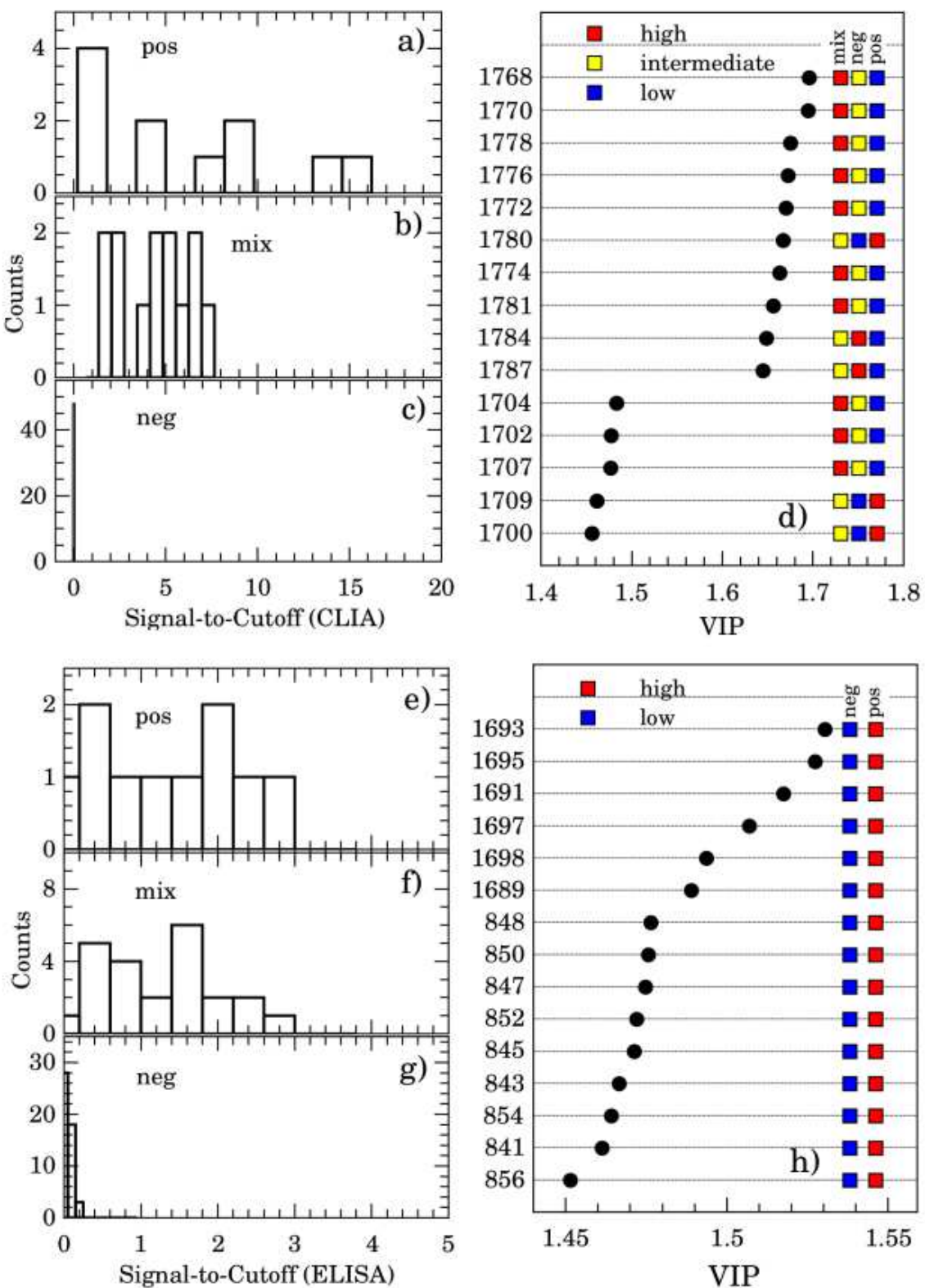

Figure 6. Histograms of Signal-to-Cutoff data of CLIA IgG antibodies against Sars-Cov-2 samples classes positive (a), mixing (b), and negative (c). Important vibrational frequencies identified by PLS-DA for three classes (positive, negative, and mixing, d) cases. Similar histograms for ELISA immunoassay are shown in e) -g). The VIP for classes discrimination is shown on $\mathrm{h}$ ). The colored boxes on the right indicate the relative intensity of the corresponding band in each group.

Obviously, the negative group presented a narrow histogram centered on 0.1 value. The outcome from the Z-test (p-value $<5 \%$ ) indicated that these IgG distributions come from distinct populations. Interestingly the histograms of Signal-to-Cutoff from ELISA (Fig. 6 e, f, and g) showed no evidence that positive and mixing groups belongs to distinct populations (p-value $>5 \%$ for $\mathrm{Z}$ test). Thus, the question that arises concerns the characteristics that gives rise to these distinct 
populations. Important vibrational bands that contribute to discrimination can help find the answer to this question.

The important vibrational frequencies identified by PLS-DA are represented in Figs. 6d) and h). For positive and negative discrimination (Fig. 6h and Table 2), $\beta$-sheet structure of Amide I of proteins $\left(1689-1698 \mathrm{~cm}^{-1}\right)$ and deoxyribose from DNA bands $\left(840-856 \mathrm{~cm}^{-1}\right)$ contributed to the greatest over-expression in the positive group. Immunoglobulins are heterodimeric proteins composed of two heavy $(\mathrm{H})$ and two light (L) chains. They can be functionally separated into variable (V) domains that bind antigens and constant $(\mathrm{C})$ domains that specify effector functions, such as complement activation or binding to $\mathrm{Fc}$ receptors[43] The basic $\mathrm{H}_{2} \mathrm{~L}_{2}$ structures consist of the Fc region (fragment, crystallizable) and Fab region (fragment, antigen binding), both composed mainly of $\beta$-pleated sheets[43].Thus the increased intensity of $\beta$-sheet band in the positive group is completely correlated with antibody recruitment in COVID-19 disease. Likewise, the increase in cellular activity due to Sars-Cov-2 inflammatory response is responsible for the increase in DNA intensity observed. However, for 3 group positive-negative-mixing discrimination, a very narrow set of vibrations confined to $1702-1785 \mathrm{~cm}^{-1}$ spectral window is the most important (Fig. 6d). These bands could be grouped in two classes: i) those overexpressed in the mix group and subexpressed in the positive group which are both related to IgG glycosilation and ii) those overexpressed in the positive group and underexpressed in mix group which are both related to thymine. As long as we can argue that the mixing group relates to a COVID-19 sub-population with highly glycosilated IgG. As all patients in the present study were oligosymptomatic, the above feature is in agreement with the Chakraborty et al. findings [13] which proposed that patients with severe COVID-19 are more likely to have $\operatorname{IgG1}$ with afucosylated Fc glycans as signature. One important point to clarify is the difference between CLIA and ELISA distribution of Signal-to-cutoff ratio (Fig. 6). It appears that the 
fluorescence yield of murine monoclonal anti-human IgG anti-body complexed to HRP of CLIA is dependent on the fucosylation of the Fc tail of human IgG.

Concerning the diagnostic performance we mention that spectral windows at $820-890,1025$ 1180, and 1685-1727 $\mathrm{cm}^{-1}$ presented excellent capability for discriminate negative and positive classes $(\mathrm{AUC}=0.80-0.86$, Fig. 7a)

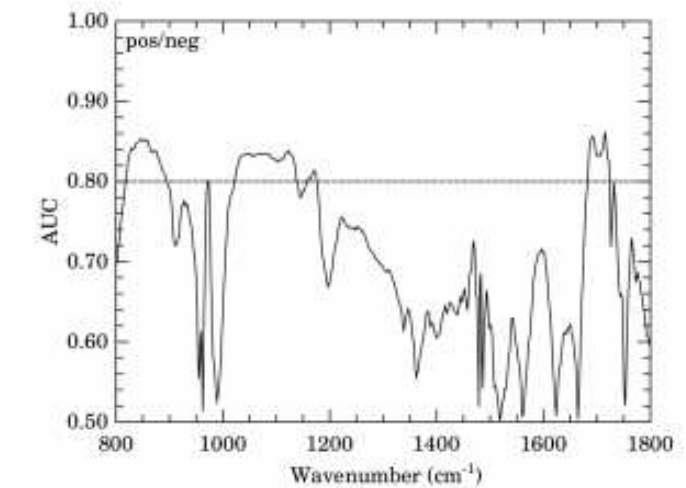

a)
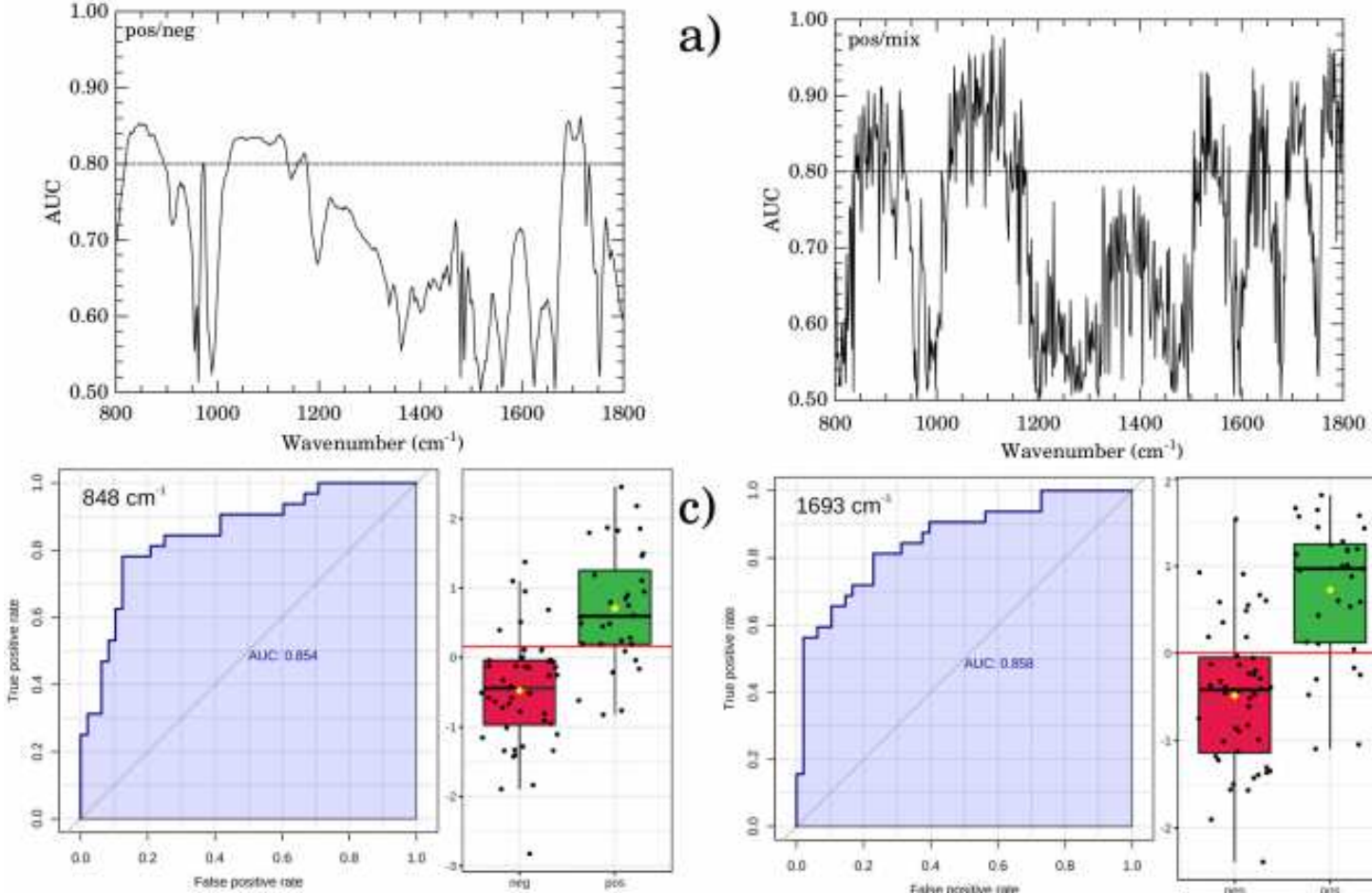

c)

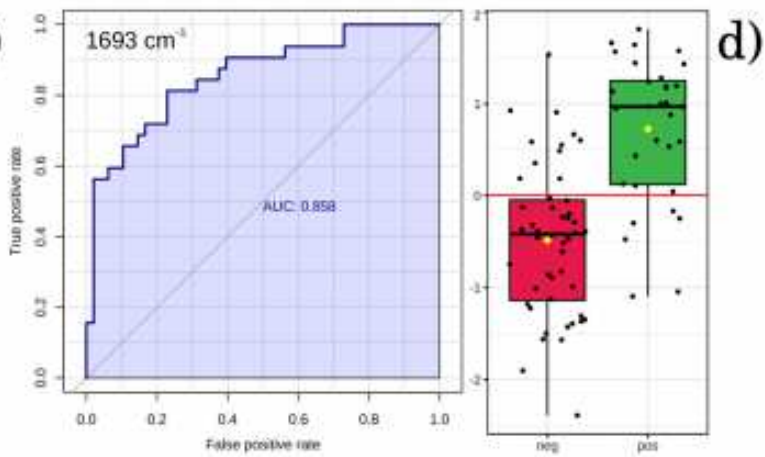

e)

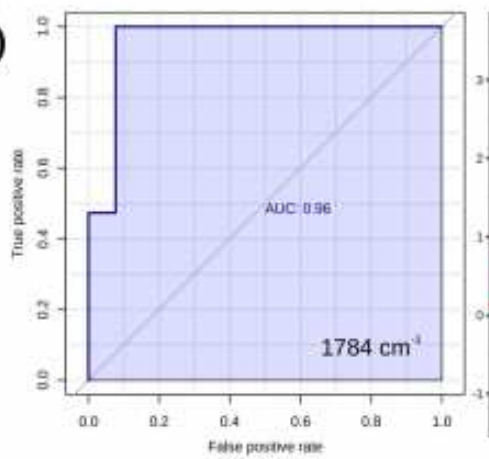

b)

d)
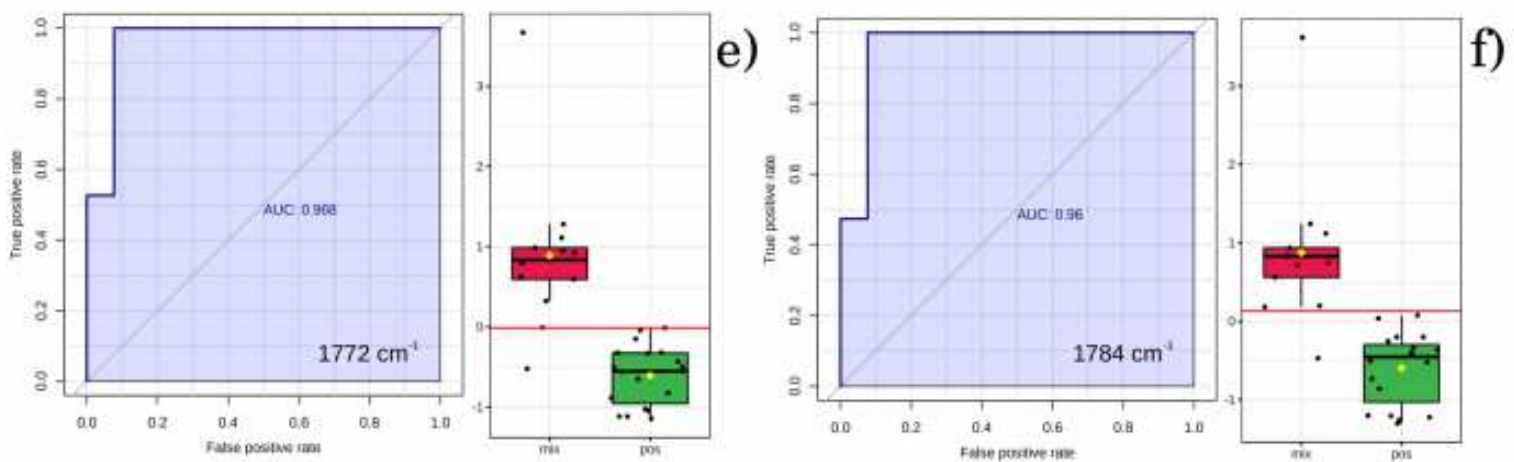

Figure 7. Area under receiver operating characteristic (AUC) against wavenumber showing those bands with excellent discriminating power (AUC > 0.80, dashed horizontal line) for positive/negative (a) and positive/mix (b) classes. Selected representative curves of receiver operating characteristic (ROC) and corresponding classification box-plot of the intensity of the left-handed helix DNA ( $\mathrm{Z}$ form) $\left(848 \mathrm{~cm}^{-1}, \mathrm{c}\right), \beta$-sheet structure of Amide I of proteins $\left(1693 \mathrm{~cm}^{-1}, \mathrm{~d}\right)$, $\mathrm{C}=\mathrm{O}$ in IgG carbonyl group (1772 and $1784 \mathrm{~cm}^{-1}$ in e) and f), respectively) bands. The horizontal red line is the threshold for classification. 
The positive and mixing classes were also discriminated in excellent grade (AUC $=0.80-$ 0.98 ) in spectral windows $850-940,1015-1170,1500-1570,1612-1652,1695-1735,1755-1790 \mathrm{~cm}^{-1}$ (Fig, 7b). This curve is comparatively nosiest respect to positive/negative case due to the low-N (only $30 \%$ of data) in this case. The representative ROC curves for negative and positive (Fig. 7 c,d) and mixing and positive (Fig. 7e,f) classes with highest AUC are also shown. The ROC curves for positive/negative (Fig. 7c,d) have binormal curve shapes while mixing/positive ones have straight line shapes due to low-N. The classification boxplots for two discriminating classes presented a significant statistical difference among groups ( $\mathrm{p}<0.05$ for $\mathrm{t}-\mathrm{Student}$ test).

\subsection{Micro-FTIR reflectance compared to other methods}

At this point is important compare our results to previous reports concerning blood testing for COVID-19. The usage of Attenuated Total Reflectance - FTIR (ATR-FTIR) for analysis of plasma from COVID-19 patients have been recently reported on literature. This technique share great similarity with our methodology and for this reason a detailed comparison with our methodology is essential.

Banerjee et al.[44] investigated a cohort of 160 clinicopathologically confirmed SARSCoV-2 patients using ATR-FTIR. They proposed a plasma processing with $75 \%$ ethanol v/v for virus inactivation followed by vortexing and drying over ATR-crystal. The reported AUC was 0.851 when considering the set of spectral and clinical (age, sex, diabetes mellitus, and hypertension) data. The discriminating performance of the spectral data alone was not reported. Zhang et al.[45] investigated the ATR-FTIR spectra of blood samples from 20 healthy donors and 76 patients, of which 41 were confirmed with COVID-19, 15 had respiratory viral infections caused by influenza A/B or respiratory syncytial virus (RSV), and 20 were with inflammation-related diseases. In this case the 
AUC for COVID-19 discrimination was reported to be 0.9947. To the best of our knowledge only these two exciting articles reports COVID-19 diagnosis using ATR-FTIR. In spite of diverse sample preparation method and amount of individuals included in the cohort, our AUC is very similar to that found by Banerjee et al. and Zhang et al. The key differences between our approach and the ATRFTIR relies on waiting time for result outcome, amount of samples which would be tested by row, and COVID-19 fatality capability prediction since we explored in deep the biochemical pieces of information in the FTIR spectra. For spectrum acquisition on ATR-FTIR a single drop of sample need the deposited and dried over the surface of ATR crystal. Then only a single patient could be tested at once. This process demands usually $>5$ hours[26]. It is not well reported whether the proteins degradation over this time would impact on quality of diagnosis. Moreover the morphological characteristics of the deposited bio-film (heterogeneity, size, wettability, dilution, among others) is dependent of drying conditions as relative moisture and temperature. The quality of spectrum (artifacts, reproducibility) is very sensible to the morphology of the bio-film. These points imposes challenges to the standardization of the method[26]. In our approach it is possible to prepare drops of several patients over the platinum substrate. Over an equivalent area of a 96 wells microplate it is possible the deposition of 500 droplets. Using the microscope it is relatively fast repositioning the IR beam over each sample which strongly increases the scale of testing of our diagnostic method. Banerjee et al. reported the fatality prediction only when considering in the statistical analysis a larger set of data including spectra and clinical pieces of information which in some aspect represent a bias in the statistical test. On the other hand we were able to clearly discriminate 3 possible groups in a independent way of validation. The clinical data were used to compare and discuss the data but not as input. 
Other essential comparison is with the gold standard method for COVID-19 testing, ELISA and also with CLIA method both used here to validate our results. Considering the test performance we will contrast our findings with some selected illustrative examples. Jagtap et al.[46] evaluated the performance of spike protein antigens for SARS-CoV-2 serology compared four spike proteins (RBD, S1, S2 and a stabilized spike trimer (ST)) using using indirect ELISA in serum from COVID19 patients and pre-2020 samples. ROC curves indicates that ST is the best candidate for serological testing with the highest area under the curve (AUC: $\mathrm{S} 1=0.86, \mathrm{~S} 2=0.90, \mathrm{RBD}=0.91, \mathrm{ST}=0.94$ ). AUC for IgG was 0.934. Huang et al.[47] compared the serological test of SARS-CoV-2 using ELISA test and reported that AUC (IgM) was 0.812 while AUC(IgG+IgM) was 0.983 . The reported AUC for CLIA anti-Sars-CoV-2 test include 0.846[48], 0.9143[49], 0.98[50], 0.831[48]. Thus we can conclude that the performance of our micro-FTIR approach for discrimination is in the same level of ELISA and CLIA.

However as summarized by Liu and Rusling[51], in general the nucleic acid-based tests against COVID-19 have reportedly suffered from a high false-negative rate which is dependent of the day of the symptom (38\% on the day, $20 \% 3$ days, and $66 \% 16$ days, after of symptom onset, respectively). Factors as sampling time, viral mutation, inadequate handling, improper storage, transportation of samples, among others had been cited to influence the result. Due their nature perform these tests require high workload, skilled personnel for testing and sample collection, special reagent kits, costly centralized infrastructure with specific equipment for process and measured the sample signal, and professional biosafety level (BSL)-2 lab[51] imposing elevated costs, relatively longer time to delivery of results (2-3 hours) and dependence of international chain of suppliers. This last aspect would be disrupted at pandemic period. One important advantage of micro-FTIR method is the minimal need of sample preparation impacting in lower cost per testing 
and relative independence of external suppliers. In our case it was a on site dilution on water followed by fast drying on ambient conditions. The time elapsed since pipetting the droplets, dry, capture spectrum and observed the result is typically $10 \mathrm{~min}$. Other aspect to mention is that ELISA and CLIA are able to detect a single protein at once. Nevertheless micro-FTIR has multiplexing advantage enabling comparison of several metabolites which increases the quality of prognosis being a suitable tool for precision medicine[52]. Oour method enable in principle probe 500 samples by row while ELISA and CLIA usually are limited to 96 samples. Table 3 summarizes the comparison between all techniques.

Table 3. Parameters for maintenance, management, operating, and performance of the main anti-Sars-Cov-2 clinical tests ELISA and CLIA compared to the micro-FTIR. Shaded boxes indicated those parameter representing advantage for micro-ATR option.

\begin{tabular}{|c|c|c|c|c|}
\hline Parameter & micro-FTIR & ATR-FTIR & ELISA & CLIA \\
\hline Sample preparation & minimal & minimal & complex & complex \\
\hline Waiting time for result & $<10 \min$ & $>5$ hours & 2-3 hours & $2-3$ hours \\
\hline $\begin{array}{l}\text { Laboratory equipment } \\
\text { requirements }\end{array}$ & intermediate & intermediate & high & high \\
\hline $\begin{array}{l}\text { Specialization of human } \\
\text { resources for usage }\end{array}$ & intermediate & intermediate & high & high \\
\hline AUC & $>0.85$ & $>0.85$ & $>0.85$ & $>0.85$ \\
\hline Multiplexing capability & yes & yes & no & no \\
\hline $\begin{array}{l}\text { COVID-19 fatality } \\
\text { prediction }\end{array}$ & yes & depends & no & no \\
\hline Reagents & free & free & expensive & expensive \\
\hline Cost of single test (US\$) & $1-10$ & $1-10$ & $50-100$ & $50-100$ \\
\hline $\begin{array}{l}\text { Cost of main equipment for } \\
\text { testing (US\$) }\end{array}$ & $20,000-50,000$ & $20,000-50,000$ & $10,000-30,000$ & $10,000-30,000$ \\
\hline $\begin{array}{l}\text { Amount of samples tested } \\
\text { by row }\end{array}$ & $>500$ & 1 & 96 & 96 \\
\hline $\begin{array}{l}\text { Dependence of international } \\
\text { logistic and supply chains }\end{array}$ & weak & weak & strong & strong \\
\hline $\begin{array}{l}\text { Operator dependence level } \\
\text { of reproducibility of } \\
\text { outcome }\end{array}$ & intermediate & intermediate & high & high \\
\hline
\end{tabular}

\section{CONCLUSIONS}

Our results showed that FTIR was able to probe key structural aspects of serum IgG from COVID-19 volunteers related to severity. The $1702-1785 \mathrm{~cm}^{-1}$ spectral window appeared to be a 
spectral marker of the degree of IgG glycosylation, allowing to probe distinctive subpopulations of COVID-19 patients, depending on their degree of severity. Furthermore, the $\beta$-sheet structure of Amide I of proteins $\left(1689-1698 \mathrm{~cm}^{-1}\right)$ and deoxyribose from DNA $\left(840-856 \mathrm{~cm}^{-1}\right)$ bands presented most significant contributions to positive and negative discrimination with a specificity of $87.5 \%$ and sensibility of $100 \%$. The computed AUC was comparable to the ELISA, CLIA, and other ATRFTIR methods (AUC > 0.85) In summary, overall discrimination of healthy and COVID-19 individuals and severity prediction could also be potentially implemented using micro-FTIR reflectance spectroscopy on blood serum samples due to direct probe of glycosilation degree of IgG.

\section{ACKNOWLEDGMENTS}

The authors would like to thank the Brazilian agencies Conselho Nacional de Desenvolvimento Científico e Tecnológico (CNPq - 311146/2015-5 and 307718/2019-0) and Fundação de Amparo à Pesquisa do Estado de São Paulo (FAPESP - 2011/19924-2) for the financial support. The authors would also thank the experimental resources provided by Multiuser Central Facilities at UFABC (CEM/UFABC).

\section{REFERENCES}

[1] Peiris, J. S. M.; Lai, S. T.; Poon, L. L. M.; Guan, Y.; Yam, L. Y. C.; Lim, W.; Nicholls, J.; Yee, W. K. S.; Yan, W. W.; Cheung, M. T.; Cheng, V. C. C.; Chan, K. H.; Tsang, D. N. C.; Yung, R. W. H.; Ng, T. K.; Yuen, K. Y. and study group , S. A. R. S. (2003). Coronavirus as a possible cause of severe acute respiratory syndrome., Lancet (London, England) 361 : 1319-1325.

[2] Huang, C.; Wang, Y.; Li, X.; Ren, L.; Zhao, J.; Hu, Y.; Zhang, L.; Fan, G.; Xu, J.; Gu, X.; Cheng, Z.; Yu, T.; Xia, J.; Wei, Y.; Wu, W.; Xie, X.; Yin, W.; Li, H.; Liu, M.; Xiao, Y.; Gao, H.; Guo, L.; Xie, J.; Wang, G.; Jiang, R.; Gao, Z.; Jin, Q.; Wang, J. and Cao, B. (2020). Clinical features of patients infected with 2019 novel coronavirus in Wuhan, China, The Lancet 395 : 497-506.

[3] (2021). World Health Organization Coronavirus (COVID-19) Dashboard, .

[4] Mehta, P.; McAuley, D. F.; Brown, M.; Sanchez, E.; Tattersall, R. S. and Manson, J. J. (2020). COVID-19: consider cytokine storm syndromes and immunosuppression, The Lancet 395 : 1033-1034. 
[5] Ruan, Q.; Yang, K.; Wang, W.; Jiang, L. and Song, J. (2020). Clinical predictors of mortality due to COVID-19 based on an analysis of data of 150 patients from Wuhan, China, Intensive Care Medicine 46 : 846-848.

[6] Fardet, L.; Galicier, L.; Lambotte, O.; Marzac, C.; Aumont, C.; Chahwan, D.; Coppo, P. and Hejblum, G. (2014). Development and Validation of the HScore, a Score for the Diagnosis of Reactive Hemophagocytic Syndrome, Arthritis \& Rheumatology 66 : 2613-2620.

[7] Abbas, A. K.; Lichtman, A. H. and Pillai, S., 2019. Basic immunology e-book: functions and disorders of the immune system. Elsevier Health Sciences, .

[8] Vidarsson, G.; Dekkers, G. and Rispens, T. (2014). IgG Subclasses and Allotypes: From Structure to Effector Functions, Frontiers in Immunology 5.

[9] Schur, P. H. (1988). IgG subclasses. A historical perspective., Monographs in allergy 23 : 1-11.

[10] Ferrante, A.; Beard, L. J. and Feldman, R. G. (1990). IgG subclass distribution of antibodies to bacterial and viral antigens., The Pediatric infectious disease journal 9 : S16-S24.

[11] Hoepel, W.; Chen, H.-J.; Geyer, C. E.; Allahverdiyeva, S.; Manz, X. D.; de Taeye, S. W.; Aman, J.; Mes, L.; Steenhuis, M.; Griffith, G. R.; Bonta, P. I.; Brouwer, P. J.; Caniels, T. G.; van der Straten, K.; Golebski, K.; Jonkers, R. E.; Larsen, M. D.; Linty, F.; Nouta, J.; van Roomen, C. P.; van Baarle, F. E.; van Drunen, C. M.; Wolbink, G.; Vlaar, A. P.; de Bree, G. J.; Sanders, R. W.; Willemsen, L.; Neele, A. E.; van de Beek, D.; Rispens, T.; Wuhrer, M.; Bogaard, H. J.; van Gils, M. J.; Vidarsson, G.; de Winther, M. and den Dunnen, J. (2021). High titers and low fucosylation of early human anti-SARS-CoV-2 IgG promote inflammation by alveolar macrophages, Science Translational Medicine 13 : eabf8654.

[12] Chakraborty, S.; Gonzalez, J.; Edwards, K.; Mallajosyula, V.; Buzzanco, A. S.; Sherwood, R.; Buffone, C.; Kathale, N.; Providenza, S.; Xie, M. M.; Andrews, J. R.; Blish, C. A.; Singh, U.; Dugan, H.; Wilson, P. C.; Pham, T. D.; Boyd, S. D.; Nadeau, K. C.; Pinsky, B. A.; Zhang, S.; Memoli, M. J.; Taubenberger, J. K.; Morales, T.; Schapiro, J. M.; Tan, G. S.; Jagannathan, P. and Wang, T. T. (2020). Proinflammatory IgG FC structures in patients with severe COVID-19, Nature Immunology 22 : 67-73.

[13] Luo, H.; Jia, T.; Chen, J.; Zeng, S.; Qiu, Z.; Wu, S.; Li, X.; Lei, Y.; Wang, X.; Wu, W.; Zhang, R.; Zou, X.; Feng, T.; Ding, R.; Zhang, Y.; Chen, Y.-Q.; Sun, C.; Wang, T.; Fang, S. and Shu, Y. (2021). The Characterization of Disease Severity Associated IgG Subclasses Response in COVID-19 Patients, Frontiers in Immunology 12.

[14] Baker, M. J.; Byrne, H. J.; Chalmers, J.; Gardner, P.; Goodacre, R.; Henderson, A.; Kazarian, S. G.; Martin, F. L.; Moger, J.; Stone, N. and Sulé-Suso, J. (2018). Clinical applications of infrared and Raman spectroscopy: state of play and future challenges, The Analyst $143: 1735-1757$.

[15] Jermyn, M.; Mok, K.; Mercier, J.; Desroches, J.; Pichette, J.; Saint-Arnaud, K.; Bernstein, L.; Guiot, M.-C.; Petrecca, K. and Leblond, F. (2015). Intraoperative brain cancer detection with Raman spectroscopy in humans, Science Translational Medicine 7 : 274ra19-274ra19. 
[16] Lin, D.; Qiu, S.; Huang, W.; Pan, J.; Xu, Z.; Chen, R.; Feng, S.; Chen, G.; Li, Y.; Short, M.; Zhao, J.; Fawzy, Y. and Zeng, H. (2018). Autofluorescence and white light imaging-guided endoscopic Raman and diffuse reflectance spectroscopy for in vivo nasopharyngeal cancer detection, Journal of Biophotonics 11 : e201700251.

[17] Mitropoulos, K.; Katsila, T.; Patrinos, G. P. and Pampalakis, G. (2018). Multi-Omics for Biomarker Discovery and Target Validation in Biofluids for Amyotrophic Lateral Sclerosis Diagnosis, OMICS: A Journal of Integrative Biology 22 : 52-64.

[18] Morais, C. L. M.; Lima, K. M. G.; Singh, M. and Martin, F. L. (2020). Tutorial: multivariate classification for vibrational spectroscopy in biological samples, Nature Protocols 15 : 2143-2162.

[19] Barauna, V. G.; Singh, M. N.; Barbosa, L. L.; Marcarini, W. D.; Vassallo, P. F.; Mill, J. G.; Ribeiro-Rodrigues, R.; Campos, L. C. G.; Warnke, P. H. and Martin, F. L. (2021). Ultrarapid On-Site Detection of SARS-CoV-2 Infection Using Simple ATR-FTIR Spectroscopy and an Analysis Algorithm: High Sensitivity and Specificity, Analytical Chemistry 93 : 2950-2958.

[20] Kitane, D. L.; Loukman, S.; Marchoudi, N.; Fernandez-Galiana, A.; Ansari, F. Z. E.; Jouali, F.; Badir, J.; Gala, J.-L.; Bertsimas, D.; Azami, N.; Lakbita, O.; Moudam, O.; Benhida, R. and Fekkak, J. (2021). A simple and fast spectroscopy-based technique for Covid-19 diagnosis, Scientific Reports 11.

[21] Wood, B. R.; Kochan, K.; Bedolla, D. E.; Salazar-Quiroz, N.; Grimley, S. L.; PerezGuaita, D.; Baker, M. J.; Vongsvivut, J.; Tobin, M. J.; Bambery, K. R.; Christensen, D.; Pasricha, S.; Eden, A. K.; Mclean, A.; Roy, S.; Roberts, J. A.; Druce, J.; Williamson, D. A.; McAuley, J.; Catton, M.; Purcell, D. F. J.; Godfrey, D. I. and Heraud, P. (2021). Infrared Based Saliva Screening Test for COVID-19, Angewandte Chemie 133 : 17239-17244.

[22] Islam, S.; Moinuddin; Mir, A. R.; Raghav, A.; Habib, S.; Alam, K. and Ali, A. (2017). Glycation, oxidation and glycoxidation of IgG: a biophysical, biochemical, immunological and hematological study, Journal of Biomolecular Structure and Dynamics 36 : 2637-2653.

[23] Joyner, M. J.; Carter, R. E.; Senefeld, J. W.; Klassen, S. A.; Mills, J. R.; Johnson, P. W.; Theel, E. S.; Wiggins, C. C.; Bruno, K. A.; Klompas, A. M.; Lesser, E. R.; Kunze, K. L.; Sexton, M. A.; Soto, J. C. D.; Baker, S. E.; Shepherd, J. R.; van Helmond, N.; Verdun, N. C.; Marks, P.; van Buskirk, C. M.; Winters, J. L.; Stubbs, J. R.; Rea, R. F.; Hodge, D. O.; Herasevich, V.; Whelan, E. R.; Clayburn, A. J.; Larson, K. F.; Ripoll, J. G.; Andersen, K. J.; Buras, M. R.; Vogt, M. N.; Dennis, J. J.; Regimbal, R. J.; Bauer, P. R.; Blair, J. E.; Paneth, N. S.; Fairweather, D.; Wright, R. S. and Casadevall, A. (2021). Convalescent Plasma Antibody Levels and the Risk of Death from Covid-19, New England Journal of Medicine 384 : 1015-1027.

[24] Padoan, A.; Bonfante, F.; Pagliari, M.; Bortolami, A.; Negrini, D.; Zuin, S.; Bozzato, D.; Cosma, C.; Sciacovelli, L. and Plebani, M. (2020). Analytical and clinical performances of five immunoassays for the detection of SARS-CoV-2 antibodies in comparison with neutralization activity, EBioMedicine 62 : 103101. 
[25] Shaffaf, T. and Ghafar-Zadeh, E. (2021). COVID-19 Diagnostic Strategies Part II: ProteinBased Technologies, Bioengineering $8: 54$.

[26] Cameron, J. M.; Butler, H. J.; Anderson, D. J.; Christie, L.; Confield, L.; Spalding, K. E.; Finlayson, D.; Murray, S.; Panni, Z.; Rinaldi, C.; Sala, A.; Theakstone, A. G. and Baker, M. J. (2020). Exploring pre-analytical factors for the optimisation of serum diagnostics: Progressing the clinical utility of ATR-FTIR spectroscopy, Vibrational Spectroscopy 109 : 103092.

[27] Varmuza, K. and Filzmoser, P., 2016. Introduction to multivariate statistical analysis in chemometrics. CRC press, .

[28] Team, R. C. and others (2013). R: A language and environment for statistical computing, .

[29] Lieber, C. A. and Mahadevan-Jansen, A. (2003). Automated Method for Subtraction of Fluorescence from Biological Raman Spectra, Applied Spectroscopy 57 : 1363-1367.

[30] Dieterle, F.; Ross, A.; Schlotterbeck, G. and Senn, H. (2006). Probabilistic Quotient Normalization as Robust Method to Account for Dilution of Complex Biological Mixtures. Application in1H NMR Metabonomics, Analytical Chemistry 78 : 4281-4290.

[31] Wehrens, R. and Mevik, B.-H. (2007). pls: Partial Least Squares Regression (PLSR) and Principal Component Regression (PCR), R package version 2.1-0, .

[32] Bijlsma, S.; Bobeldijk, I.; Verheij, E. R.; Ramaker, R.; Kochhar, S.; Macdonald, I. A.; van Ommen, B. and Smilde, A. K. (2005). Large-Scale Human Metabolomics Studies: A Strategy for Data (Pre-) Processing and Validation, Analytical Chemistry 78 : 567-574.

[33] Szymańska, E.; Saccenti, E.; Smilde, A. K. and Westerhuis, J. A. (2011). Double-check: validation of diagnostic statistics for PLS-DA models in metabolomics studies, Metabolomics 8 : 316.

[34] Zou, K. H.; O'Malley, A. J. and Mauri, L. (2007). Receiver-Operating Characteristic Analysis for Evaluating Diagnostic Tests and Predictive Models, Circulation 115 : 654-657.

[35] Ma, H.; Bandos, A. I. and Gur, D. (2014). On the use of partial area under the ROC curve for comparison of two diagnostic tests, Biometrical Journal 57 : 304-320.

[36] Justel, A.; Peña, D. and Zamar, R. (1997). A multivariate Kolmogorov-Smirnov test of goodness of fit, Statistics \& Probability Letters 35 : 251-259.

[37] Markowski, C. A. and Markowski, E. P. (1990). Conditions for the Effectiveness of a Preliminary Test of Variance, The American Statistician 44 : 322.

[38] Sanyaolu, A.; Okorie, C.; Marinkovic, A.; Patidar, R.; Younis, K.; Desai, P.; Hosein, Z.; Padda, I.; Mangat, J. and Altaf, M. (2020). Comorbidity and its Impact on Patients with COVID19, SN Comprehensive Clinical Medicine 2 : 1069-1076.

[39] Buijs, J.; Norde, W. and Lichtenbelt, J. W. T. (1996). Changes in the Secondary Structure of Adsorbed IgG and F(ab ')2 Studied by FTIR Spectroscopy, Langmuir 12 : 1605-1613. 
[40] Movasaghi, Z.; Rehman, S. and ur Rehman, D. I. (2008). Fourier Transform Infrared (FTIR) Spectroscopy of Biological Tissues, Applied Spectroscopy Reviews 43 : 134-179.

[41] Ahmad, S.; Moinuddin; Khan, R. H. and Ali, A. (2012). Physicochemical studies on glycation-induced structural changes in human IgG, IUBMB Life 64 : 151-156.

[42] Krebs, H. A. (1950). Chemical Composition of Blood Plasma and Serum, Annual Review of Biochemistry 19 : 409-430.

[43] Schroeder, H. W. and Cavacini, L. (2010). Structure and function of immunoglobulins, Journal of Allergy and Clinical Immunology 125 : S41-S52.

[44] Banerjee, A.; Gokhale, A.; Bankar, R.; Palanivel, V.; Salkar, A.; Robinson, H.; Shastri, J. S.; Agrawal, S.; Hartel, G.; Hill, M. M. and Srivastava, S. (2021). Rapid Classification of COVID-19 Severity by ATR-FTIR Spectroscopy of Plasma Samples, Analytical Chemistry 93 : 10391-10396.

[45] Zhang, L.; Xiao, M.; Wang, Y.; Peng, S.; Chen, Y.; Zhang, D.; Zhang, D.; Guo, Y.; Wang, X.; Luo, H.; Zhou, Q. and Xu, Y. (2021). Fast Screening and Primary Diagnosis of COVID-19 by ATR-FT-IR, Analytical Chemistry 93 : 2191-2199.

[46] Jagtap, S.; Ratnasri, K; Valloly, P.; Sharma, R.; Maurya, S.; Gaigore, A.; Ardhya, C.; Biligi, D. S.; Desiraju, B. K.; Natchu, U. C. M.; Saini, D. K. and Roy, R. (2021). Evaluation of spike protein antigens for SARS-CoV-2 serology, Journal of Virological Methods 296 : 114222.

[47] Huang, S.; Lin, C.; Yan, M.; Li, H.; Liu, T.; Michael, W.; Xiang, J. and Shen, C. (2020). Rapid Detection of COVID-19 by Serological Methods and the Evaluation of Diagnostic Efficacy of IgM and IgG, Clinical Laboratory 66.

[48] Soleimani, R.; Khourssaji, M.; Gruson, D.; Rodriguez-Villalobos, H.; Berghmans, M.; Belkhir, L.; Yombi, J.-C. and Kabamba-Mukadi, B. (2020). Clinical usefulness of fully automated chemiluminescent immunoassay for quantitative antibody measurements in COVID-19 patients, Journal of Medical Virology 93 : 1465-1477.

[49] Mendoza, R.; Silver, M.; Zuretti, A.; Christian, M.; Das, B.; Norin, A.; Borgen, P.; Libien, J. and Bluth, M. (2021). Correlation of Automated Chemiluminescent Method with Enzyme-Linked Immunosorbent Assay (ELISA) Antibody Titers in Convalescent COVID-19 Plasma Samples: Development of Rapid, Cost-Effective Semi-Quantitative Diagnostic Methods, Journal of Blood Medicine Volume 12 : 157-164.

[50] Fabián, C. L. R. and Briceño, L. U. (2020). Anti-SARS-Cov-2 IgA in Current Scenario of IgM and IgG Rapid Test: a New Alternative for the Diagnostic of COVID-19, SN Comprehensive Clinical Medicine 2 : 2167-2169.

[51] Liu, G. and Rusling, J. F. (2021). COVID-19 Antibody Tests and Their Limitations, ACS Sensors $6:$ 593-612. 
[52] Zhou, A.; Sabatello, M.; Eyal, G.; Lee, S. S.-J.; Rowe, J. W.; Stiles, D. F.; Swanson, A. and Appelbaum, P. S. (2021). Is precision medicine relevant in the age of COVID-19?, Genetics in Medicine 23 : 999-1000. 